\title{
COBERTURA MÉDICA DE UNA PERSONA ADULTA MAYOR: RAZONES HUMANITARIAS POR SOBRE CUESTIONES ADMINISTRATIVAS
}

\author{
MEDICAL COVERAGE OF AN ELDERLY PERSON: \\ HUMANITARIAN REASONS FOR ADMINISTRATIVE \\ MATTERS
}

\section{María Guadalupe Neder}

DOI: https://doi.org/10.37767/2591-3476(2020)31

\section{Comentario a}

Incidente de medida cautelar de Lenzano, Luis Eduardo, OSPOSE y ANSES en Autos Lenzano, Luis Eduardo, OSPOSE y ANSES s/amparo ley 16.986

\section{Cámara Federal de Mendoza, Sala "A"}

(Poder Judicial de la Nación)

\author{
Disponible en \\ https://bit.ly/2EezZea
}

\section{RESUMEN:}

A través del presente comentario se intentará hacer una breve síntesis del razonamiento de la justicia federal mendocina en una ejemplar decisión que recayó sobre una persona adulta mayor. El tribunal interviniente teniendo en cuenta la elevada edad del amparista, su condición de jubilado y su estado de incertidumbre como consecuencia de la actitud desplegada por la obra social, lo considero merecedor de una tutela preventiva y resolvió mantener su afiliación a la obra social pese haber adquirido su condición de jubilado.

\begin{abstract}
Through this comment, an attempt will be made to summarize the reasoning of the federal justice system in Mendoza in an exemplary decision that fell on an elderly person. The intervening court, taking into account the high age of the amparo, his retirement status and his state of uncertainty as a consequence of the attitude displayed by the social work, considered him deserving of preventive protection and decided to maintain his affiliation to the social work despite having acquired his retirement status.
\end{abstract}

1 Abogada (UNC); Maestranda en Derecho Procesal (Universidad Siglo 21); miembro de los Proyectos de investigación "Justicia y protección de las personas en condición de vulnerabilidad" y "Retos normativos para el correcto y seguro desarrollo de la telemedicina en la Provincia de Córdoba" de la Sala de Derecho a la Salud del Instituto de Investigación en Ciencias Jurídicas (IDI-CJ), Universidad Blas Pascal (UBP). Se desempeña como auxiliar del Juzgado Civil y Comercial de 12 Nominación de la Provincia de Córdoba. Mail: mariaguadalupeneder@gmail.com. ORCID iD: https://orcid.org/0000-0002-0857-4127 
PALABRAS CLAVE: Persona adulta mayor; derecho a la salud; medida cautelar; perspectiva de vulnerabilidad; tutela efectiva diferenciada.

KEY WORDS: Elderly person; right to health; caution; vulnerability perspective; differentiated effective guardianship.

\section{Introducción}

En el mes de septiembre del año 2019, en los autos caratulados "Incidente de medida cautelar de L., L. E. OSPOCE y ANSES en autos L., L. E. c/ OSPOCE y ANSES s/amparo ley 16.986", la Cámara Federal de Mendoza - Sala "A" resolvió confirmar la resolución del Juzgado Federal de Mendoza $n^{\circ} 2$ mediante el cual se declaró procedente la medida cautelar solicitada por la parte actora consistente en mantener su afiliación a la obra social pese haber adquirido su condición de jubilado.

\section{El caso}

La resolución judicial fue apelada por la Obra Social del Personal del Organismo de Control Externo (OSPOCE) -parte demandada- por considerarse agraviada en dos aspectos: en primer lugar, considero que la resolución judicial no puede hacerse efectiva por un impedimento legal; $y$ en segundo lugar, sostuvo que resulta de cumplimiento imposible.

Con relación al primer agravio, manifestó que por resolución de ANSES ( $N^{\circ}$ 684/97) la opción del pasivo sólo podrá efectuarse ante las obras sociales que se hayan inscripto en el Registro de Agente del Sistema Nacional del Seguro de Salud para la Atención Médica de Jubilados y Pensionados (Dec. No 292/95 y 492/95) y, en consecuencia, atento a que la OSPOCE no se encuentra inscripta en el registro referido, no posee habilitación para incorporar dentro de su población beneficiaria a jubilados y pensionados.

Con relación al segundo agravio, la demandada manifestó que resulta de cumplimiento imposible ya que el plan que se le ordena brindar al actor es un plan que la obra social no ofrece, que nunca lo brindo, y que es la entidad Swiss Medical la que lo gestiona.

\section{Resolución del tribunal ad quem}

La Cámara Federal de Mendoza consideró que no corresponde hacer lugar al remedio incoado por la demandada y confirmó la medida cautelar ordenada por el juez. Para así resolver, tuvo en cuenta los fundamentos de hecho y de derecho que se detallan a continuación:

1) Con respecto al primer agravio (incorrecta interpretación de los alcances de la normativa legal aplicable al caso) la Cámara, previo a efectuar un análisis de procedencia de la medida cautelar solicitada, hizo hincapié en el ordenamiento jurídico vigente en la materia.

En primer lugar, sostuvo que, conforme al rango constitucional que posee el derecho a la salud, hay que atender los términos de la pretensión, a fin de garantizar su protección, y, además, hay que exigir a los órganos judiciales que efectúen una interpretación extensiva (y no restrictiva) de su procedencia con el objeto de no tornar ilusoria su aplicación.

En dicho marco, explico que el valor vida involucrado y la dignidad de la persona resultan ser un objetivo que prima por sobre otros aspectos secundarios que condicionen el cum- 
plimiento del deber de brindar satisfactoria, oportuna y eficiente respuesta a la atención que se requiere.

Por su parte, destaco que es reiterada la doctrina y jurisprudencia que sostiene que el derecho a la vida comprende a la dignidad, la calidad de vida, y el goce del ser humano. En este sentido, ejemplifico que las patologías son contingencias en la vida de las personas que afectan su calidad de vida y en la medida que pueden ser aliviadas o sanadas deben serlo, pues si no incorporan un sufrimiento que poco a poco denigra esa calidad de vida humana.

En segundo lugar, efectuó un análisis de los presupuestos que debe reunir el otorgamiento de la medida cautelar solicitada, haciendo hincapié en tres aspectos importantes:

a) Naturaleza de las medidas cautelares: examen de verosimilitud y no de certeza del derecho pretendido.

Explico que las medidas precautorias no exige a los magistrados el examen de certeza sobre la existencia del derecho pretendido, sino solo de su verosimilitud, y que el juicio de verdad en esta materia se encuentra en oposición a la finalidad del instituto cautelar, que no es otra cosa que atender a aquello que no exceda del marco de lo hipotético, dentro del cual, asimismo, agota su virtualidad.

b) Verosimilitud del derecho invocado: se encuentra acreditado en autos que el actor es afiliado a la empresa de medicina pre paga

Con relación a este requisito, el tribunal destacó que se refiere a la posibilidad de que el derecho exista y no a una incontestable realidad, la cual sólo se logrará al agotarse el trámite. Señaló que la cautelar otorgada a favor del amparista tuvo por finalidad impedir que el derecho cuyo reconocimiento se pretende, pierda eficacia durante el tiempo que dure el proceso.

Estimó que este requisito está se encuentra acreditado atento la calidad de afiliado a la empresa de medicina pre paga de la que gozaba el amparista, hecho que no fue negado por la demandada.

Con respecto a la legislación vigente, sostuvo que la ley de obras sociales n²3.660 (art. $8^{\circ}$ y decreto reglamentario 576/93), confirma que la mera circunstancia de obtener la jubilación no implicaba sin más la transferencia del beneficiario al Instituto Nacional de Servicios Sociales para Jubilados y Pensionados (INSSJP), sino que subsistía para el ex trabajador el derecho de permanecer en la obra social que le prestaba servicios hasta entonces; conclusión que, a su vez, fue ratificada por el art. 20 de la ley 23.660 y su norma reglamentaria, al disponer que cuando el afiliado escogiese un agente de seguro distinto del INSSJP, éste debería transferir, en igual plazo, el monto equivalente al costo de módulo de Régimen de Atención Médica Especial para Pasivos, que se garantiza a todos los jubilados y pensionados.

En particular, sostuvo que el art. 10, inc. c, de la ley de obras sociales dispone que el carácter de beneficiario, subsistirá mientras se mantenga el contrato de trabajo o la relación de empleo público y el trabajador o empleado reciba remuneración del empleador, salvo en el supuesto de extinción del contrato de trabajo, en cuyo caso los trabajadores que se hubiesen desempeñado en forma continuada durante más de tres meses, man- 
tendrán su calidad de beneficiarios durante un período de tres meses, contados desde su distracto, sin obligación de efectuar aportes (inc. a).

En ese contexto normativo, la Cámara concluyo que se debe interpretar que el distracto que contempla la norma no es el que tiene lugar con motivo de la jubilación del trabajador (lo que acontece en el caso de autos), sino el que se verifica por otras circunstancias, como son las previstas en los distintos incisos del artículo, pues de otro modo quedaría sin contenido el art. 8 de la ley 23.660, en cuanto establece en su inc. b), con carácter general, que quedan obligatoriamente incluidos en calidad de beneficiarios los jubilados.

Por último, con respecto a la alegación de la accionada en cuanto a la imposibilidad para la actora, de optar por la obra social accionada, con motivo de que no se encuentra inscripta en el registro de prestadores creado por los decretos 292 y 492 ambos del año 1995, cabe reiterar que el derecho de la accionante a las prestaciones médico asistenciales que le corresponden por su carácter de afiliada con una antigüedad ininterrumpida de más de diez (10) años, circunstancia que no ha sido negada radica en el vínculo de origen que los une, y no en la opción que prevén dichas normas 0 en la Resolución Conjunta № 151/95 MS y № 364/95 MTSS.

c) Peligro en la demora: elevada edad del amparista, su condición de jubilado y su estado de incertidumbre como consecuencia de la actitud desplegada por la obra social

Finalmente, en cuanto al peligro en la demora subrayo que encontrándose involucrado el derecho a la salud, de no brindarse tutela expedita y anticipada, aún a título provisorio, la protección estatal podría "llegar tarde" ya que de nada serviría continuar con las actuaciones hasta obtener certezas e incluso, de corresponder, aplicar sanciones, puesto que para entonces la salud del peticionante, podría sufrir consecuencias irreversibles.

Entendió que este requisito se encuentra suficientemente acreditado "en atención a la elevada edad del amparista, su condición de jubilado y su estado de incertidumbre como consecuencia de la actitud desplegada por la obra social, lo que merece una tutela preventiva".

Fundamento su postura en jurisprudencia al respecto, al sostener que la urgencia de la medida guarda una relación directa con la verosimilitud del derecho, que en el caso se aprecia no como un emergente del análisis complejo al hecho y prueba, sino de una flagrante violación constitucional, ello con la provisoriedad que encierra el juicio de valor en una resolución cautelar. Agrego que "Ios requisitos para la procedencia genérica de las medidas cautelares se hallan relacionadas entre sí de tal modo, que a mayor verosimilitud del derecho cabe no ser tan exigentes en la gravedad e inminencia del daño y viceversa, cuando existe riesgo de un daño de extrema gravedad e irreparabilidad, el rigor acerca del fumus se puede atenuar (Cámara Federal Capital, LL, 1984A, p. 265 y 459)".

En definitiva, la Sala consideró que los argumentos aportados por la recurrente deben ser objeto de debate y prueba en la etapa respectiva, por lo que corresponde otorgar en forma precautoria supremacía al derecho de acceder al sistema de salud, es decir, que "existen prioritarias razones humanitarias por sobre cuestiones administrativas que habrían motivado la denunciada 'baja automática' del actor".

2) Por último, con respecto al segundo agravio (brindar al actor un plan que la demandada no ofrece), la Cámara sostuvo que cabe desestimarlo ya que el resolutivo 
en crisis ordena restablecer la afiliación en las mismas condiciones existentes al mes de octubre de 2018, sin hacer referencia a algún plan distinto del que era beneficiario el amparista.

\section{Una resolución judicial a la luz de las 100 Reglas de Brasilia y de la Convención In- teramericana sobre Protección de los Derechos Humanos de las Personas Mayores}

Luego de haber efectuado una breve síntesis del caso, se infiere que la resolución del Juzgado y de la Cámara interviniente son ajustadas a derecho y constituyen una clara manifestación de una verdadera tutela judicial efectiva que debe brindarse a los justiciables en situación de vulnerabilidad, en especial, a las personas adultas mayores.

A pesar de que la resolución de la Cámara no haya hecho expresa mención de los postulados de las 100 Reglas de Brasilia sobre Acceso a la Justicia de las personas en condición de vulnerabilidad ${ }^{2}$ y de la Convención Interamericana sobre derechos de las Personas Mayores ${ }^{3}$, su actuar se ajustó a tales preceptos.

En primer lugar, se pude decir que la resolución fue dictada con perspectiva de vulnerabilidad. En nuestro derecho interno, si bien el Código Civil y Comercial de la Nación (CCCN) no contempla una protección específica y expresa de las personas en condición de vulnerabilidad ${ }^{4}$, si lo hace su proyecto de reforma ya que una de las propuestas más destacadas es la incorporación de una figura jurídica trascendental como lo es la protección jurídica diferenciada de personas que se encuentran en situación de vulnerabilidad. En función de ello, se propone incorporar un segundo párrafo al actual art. 515, el cual quedaría redactado en los siguientes términos: "Personas en condición de vulnerabilidad. Las personas en condición de vulnerabilidad tienen derecho a una tutela efectiva diferenciada, que promueva, proteja y asegure el pleno y eficaz goce de sus derechos y el respeto de su dignidad. Se considera en condición de vulnerabilidad aquella persona que, en razón de su edad, género, estado físico o mental, o por circunstancias sociales, económicas, étnicas o culturales, encuentre especiales dificultades para el efectivo ejercicio y reconocimiento de los derechos de los que es titular".

De esta postura se hizo eco la propia Corte Interamericana de Derechos Humanos (CIDH) al señalar que: “(...) toda persona que se encuentre en una situación de vulnerabilidad es titular de una protección especial, en razón de los deberes especiales cuyo cumplimiento por parte del Estado es necesario para satisfacer las obligaciones generales de respeto y garantía de los derechos humanos. La Corte reitera que no basta que los Estados se abstengan de violar los derechos, sino que es imperativa la adopción de medidas positivas, determinables en función de las particulares necesidades de protección del sujeto de derecho, ya sea por su condición personal o por la situación específica en que se encuentre $(. . .)^{\prime \prime 6}$.

\footnotetext{
2 Actualización aprobada por la Asamblea Plenaria de la XIX edición de la Cumbre Judicial Iberoamericana, abril de 2018, Quito-Ecuador.

3 La ley nacional n 27.360 (9 de mayo de 2017) aprueba la Convención Interamericana sobre Protección de los Derechos Humanos de las Personas Mayores, adoptada por la Organización de los Estados Americanos durante la 45a Asamblea General de la OEA, el 15 de junio de 2015.

4 Puede encontrarse una aproximación en lo regulado en materia de procesos de familia, en especial en el art. 706 inc. a) que prevé que las normas que rigen el procedimiento deben ser aplicadas de modo de facilitar el acceso a la justicia, especialmente tratándose de personas vulnerables, y la resolución pacífica de los conflictos.

5 Art. 51.- Inviolabilidad de la persona humana. La persona humana es inviolable y en cualquier circunstancia tiene derecho al reconocimiento y respeto de su dignidad.

6 Corte Interamericana de Derechos Humanos, sentencia de fecha 4 de julio de 2006, Caso Ximenes Lopes VS. Brasil.
} 
Sin perjuicio de ello, mediante Acordada n 5 del año 2009 la Corte Suprema de Justicia de la Nación (CSJN) resolvió adherirse a las 100 Reglas de Brasilia sobre acceso a la justicia de las personas en condición de vulnerabilidad, y en consecuencia de ello, los jueces y juezas de la República Argentina deben seguirlas -en cuanto resulte procedente- como guía en los asuntos a que se refieren.

Las 100 Reglas de Brasilia se caracterizan por ser el instrumento normativo que otorga mayor contención jurídica a las personas en situación de vulnerabilidad. La adhesión a las mismas trajo como principal consecuencia el cambio de paradigma en el abordaje sustancial y procesal, de las causas judiciales en las que se encuentran implicados reclamos o derechos de justiciables en estado de vulnerabilidad, particularmente de las personas adultas mayores.

Conforme las mismas, uno de los principales grupos vulnerables son aquellas personas que en razón de su edad encuentran especiales dificultades para el efectivo ejercicio y reconocimiento de los derechos de los que son titular (regla 4). En este sentido, establece que el envejecimiento también puede constituir una causa de vulnerabilidad cuando la persona adulta mayor encuentre especiales dificultades, atendiendo a sus capacidades funcionales y/o barreras producto del entorno económico y social, para ejercitar sus derechos ante el sistema de justicia, con pleno respeto a su dignidad (regla 6).

La importancia de las 100 Reglas radica en que, a través suyo, se visibiliza una necesidad que debe estar presente en todos los operadores judiciales: el reconocimiento no solo formal sino material de los derechos. Esto ha quedado plasmado en la exposición de motivos, al sostener que: "El sistema judicial se debe configurar, y se está configurando, como un instrumento para la defensa efectiva de los derechos de las personas en condición de vulnerabilidad. Poca utilidad tiene que el Estado reconozca formalmente un derecho si su titular no puede acceder de forma efectiva al sistema de justicia para obtener la tutela de dicho derecho" y por ello, la recomendación principal que hacen las Reglas es tener "una actuación más intensa para vencer, eliminar o mitigar dichas limitaciones" $y$, de esta manera, el Poder Judicial puede convertirse en un gran instrumento de transformación social reduciendo las desigualdades sociales y colaborando con la unión de la sociedad.

Sobre este punto, la doctrina tiene dicho que el envejecimiento puede constituir una causa de vulnerabilidad, cuando la población adulta mayor -atendiendo a sus capacidades funcionales para ejercitar sus derechos ante el sistema de justicia- se encuentre en situación de posible desprotección o fragilidad; buscando garantizar la eficacia de sus derechos y lograr una igualdad real. Al respecto las 100 Reglas de Brasilia sobre el Acceso de Justicia de las personas en condiciones de vulnerabilidad nos habla de "...repensar su condición de sujeto de derecho a fin de situarle ante la ley realmente en igual posición que los demás... necesidad de fortalecer el reconocimiento de sus derechos...como verdaderos derechos subjetivos" (Marzioni, Cóceres y Gazzo Boero, 2016: 90)

En segundo lugar, el instrumento normativo especial de protección de este grupo vulnerable es la Convención Interamericana sobre derechos de las Personas Mayores, integrativa del bloque de constitucionalidad que atraviesa todo nuestro ordenamiento jurídico, en función de lo expresamente dispuesto por el art. 75 inc. 22 de la Constitución Nacional (CN). 
La Convención considera "persona mayor" a aquella de 60 años o más, salvo que la ley interna determine una edad base mayor o menor, siempre que esta no sea superior a los 65 años. Concepto que incluye, entre otros, el de persona adulta mayor (artículo2). Es obligación del Estado adoptar todas las medidas necesarias a fin de garantizarles un trato diferenciado y preferencial en todos los ámbitos, incluido un adecuado acceso a la justicia (artículo 4, apartado c).

En cuanto a las personas mayores, en verdad la protección no debería derivarse de un simple cómputo aritmético, por el hecho de haber alcanzado una determinada edad; no hay un tiempo fijo que ineludiblemente marque el ingreso a la ancianidad. En todo caso, será menester considerar en cada supuesto concreto si el envejecimiento constituye una causa de vulnerabilidad cuando la persona adulta mayor se encuentre en especiales dificultades, atendiendo a sus capacidades funcionales, para ejercitar sus derechos ante el sistema de justicia (Berizonce, 2018: 846).

Ahora bien, en lo que aquí nos interesa su art. 31 garantiza el derecho de acceso a la justicia y establece que los Estados Partes se comprometen a asegurar que la persona mayor tenga acceso efectivo a la justicia en igualdad de condiciones con las demás, incluso mediante la adopción de ajustes de procedimiento en todos los procesos judiciales y administrativos en cualquiera de sus etapas. Asimismo, se garantiza la debida diligencia y el tratamiento preferencial a la persona mayor para la tramitación, resolución y ejecución de las decisiones en procesos administrativos y judiciales, advirtiendo que la actuación judicial deberá ser particularmente expedita en casos en que se encuentre en riesgo la salud o la vida de la persona mayor.

En este marco, se puede observar que por imperio de los postulados de las 100 Reglas y de la mencionada Convención, las personas adultas mayores merecen una especial protección, a través de una tutela sustancial y procesal efectiva diferenciada.

La CIDH se hizo eco de ello en el destacado caso Poblete Vilches y otros vs. Chile7, en el cual se pronunció por primera vez respecto del derecho autónomo a la salud, como parte integrante de los derechos económicos, sociales, culturales y ambientales e introduce claramente el principio de que el paciente es una persona vulnerable. Con relación a las personas de elevada edad, resaltó la importancia de visibilizar a las personas mayores como sujetos de derechos con especial protección y por ende de cuidado integral, con el respeto de su autonomía e independencia. Considero que respecto de las personas adultas mayores, como grupo en situación de vulnerabilidad, existe una obligación reforzada de respeto y garantía de su derecho a la salud y que ello se traduce en la obligación de brindarles las prestaciones de salud que sean necesarias de manera eficiente y continua. En consecuencia, el incumplimiento de dicha obligación surge cuando se les niega el acceso a la salud o no se garantiza su protección, pudiendo también ocasionar una vulneración de otros derechos.

Del marco normativo reseñado, se puede concluir que tener por acreditado el requisito de peligro en la demora teniendo en cuenta la elevada edad del amparista, su condición de jubilado, su estado de incertidumbre como consecuencia de la actitud desplegada por la obra social, y hacerlo merecedor de una tutela preventiva, es prueba evidente de que

7 Sentencia de la CIDH de fecha 08.03.18. 
los tribunales mendocinos hicieron eco de los postulados al que hicimos referencia en los párrafos anteriores.

Finalmente, con respecto a los requisitos de procedencia de las medidas cautelares cuando están en juego derechos de personas en situación de vulnerabilidad, es importante destacar el pronunciamiento de nuestro Tribunal Superior de Justicia de la Provincia de Córdoba (TSJ), mediante el cual sostuvo que en los casos de alta vulnerabilidad, dichos requisitos deben flexibilizarse, ya que deben priorizarse la situación de los peticionantes. Así se ha dicho que "...el análisis de los requisitos para la procedencia de una medida cautelar debe ser resignificado y efectuado con el criterio más amplio posible en términos protectorios cuando se trata de un afiliado con una discapacidad acreditada, circunstancia que lo vuelve una persona especialmente vulnerable. En efecto, en estos casos, tal como lo ha expresado este TSJ, no debe perderse de vista 'la perspectiva de la vulnerabilidad' bajo cuyo prisma debe ensayarse cualquier salida jurisdiccional vinculada con personas en situación de vulnerabilidad; esto es, 'personas que, por razón de su edad, género, estado físico o mental, o por circunstancias sociales, económicas, étnicas y/o culturales, encuentran especiales dificultades para ejercitar con plenitud ante el sistema de justicia los derechos reconocidos por el ordenamiento jurídico (100 Reglas de Brasilia sobre Acceso a la Justicia de las Personas en Condición de Vulnerabilidad, sección segunda, 1.3)" .

\section{Conclusiones}

La Convención Interamericana sobre derechos de las Personas Adultas Mayores, las 100 Reglas de Brasilia y el CCCN (e incluso su proyecto de reforma) impulsan grandes desafíos para los operadores judiciales, en especial a la magistratura, a la hora de hacer cumplir efectivamente sus postulados y promover el reconocimiento no solo formal sino también material de los derechos de las personas que se encuentran en situación de vulnerabilidad, en especial, en razón de su edad.

Concretamente, implica una sensibilización en los funcionarios judiciales y magistrados, dictando resoluciones con "perspectiva de vulnerabilidad", prisma que debe ensayarse ante cualquier salida jurisdiccional vinculada con personas en situación de vulnerabilidad. Ello implica que, por encontrarse en juego derechos fundamentales de alto valor jurídico, en ciertos casos puedan aceptarse simplificaciones de procesos y flexibilizaciones en las formas, sin que ello implique una pérdida de certeza respecto del debido proceso.

En el supuesto bajo análisis, los jueces intervinientes al resolver declarar procedente la medida cautelar solicitada por la parte actora, en base a razones humanitarias y no administrativas, protegieron no solo formal sino materialmente su derecho. Como consecuencia de ello, se desprende que la edad es una causal de vulnerabilidad y que acredita un peligro en la demora y más aún cuando están en juego derechos del más alto valor, como la salud. 


\section{REFERENCIAS BIBLIOGRÁFICAS}

- Berizonce, Roberto Omar (2018). Regulación procesal de las tutelas diferenciadas de la Constitución. Revista Anales de la Facultad de Ciencias Jurídicas y Sociales. Universidad Nacional de La Plata. UNLP. Año 15/Nº 48-2018. Anual. Impresa ISSN 0075-7411-Electrónica ISSN 2591-6386. Disponible en: https://revistas.unlp.edu.ar/RevistaAnalesJursoc/ article/download/6005/5366/

- Marzioni, Cristina Ester; Cóceres María Celeste; y Gazzo Boero María Virginia. Transformaciones jurídicas y sociales en el nuevo Código Civil y Comercial de la Nación Argentina para los adultos mayores. Revista de la Facultad de Ciencias Jurídicas y Sociales, Universidad Nacional del Litoral (UNL). Disponible en: https://bibliotecavirtual.unl.edu.ar/publicaciones//index.php/NuevaEpoca/article/download/6231/9208. 